\title{
Compound Salvia Miltiorrhiza Preparation for the Treatment of Retinal Vein Occlusion: A Systematic Review
}

Hui Wang, Hongcai Shang*, Junhua Zhang and Wei Mu

Tianjin University of Traditional Chinese Medicine, Tianjin, China

\begin{abstract}
Objective: To evaluate the efficacy and safety of compound salvia miltiorrhiza preparation for retinal vein occlusion.

Methods: We searched the Cochrane library, Medline, Embase, CBM, CNKI, VIP and the Wanfang database (data updated to August, 2011) for relevant clinical research on compound salvia miltiorrhiza preparation for retinal vein occlusion.

Results: A total of two studies involving 137 patients met the inclusion criteria for this review. Results of the two studies were not pooled because significant heterogeneity was found between them. Data analysis showed that RR and $95 \% \mathrm{Cl}$ for both studies were $1.12[0.92,1.36]$ and $1.40[0.95,2.07]$ respectively and the $P$ value was 0.04 . As a result, there was no statistically significant difference between the curative effects of the two interventions. In one study, "days needed for patients to experience improved symptoms of fundus hemorrhage and edema" was recorded and adopted as an observational indicator. Analysis of the data for "recovery days for total stem occlusion" showed compound salvia miltiorrhiza injection had a better effect than control group (WMD -15, 95\% Cl [- 25.74, - 4.26], P = 0.006). However, analysis of the data for another two indicators, "recovery days for branch occlusion" and "improvement in retinal edema" suggested that no significant statistical difference was observed between the two interventions $(-2.00[-4.49$, 0.49], $\mathrm{P}=0.12$; WMD $0.90,95 \% \mathrm{Cl}[-1.92,3.72], \mathrm{P}=0.53$ ). Moreover, one study took "improvement in the patients' visua acuity" as a measure to test efficacy. And our analysis found that there was still no statistically significant difference between the two groups (WMD $0.10,95 \% \mathrm{Cl}[-0.11,0.31], \mathrm{P}=0.34$ ).

Conclusion: The results suggested that compound salvia miltiorrhiza preparation is more efficacious and safer to use than Urokinase in improving symptoms of total stem occlusion and fundus hemorrhage. However, the efficacy and safety of compound salvia preparation in treating retinal vein occlusion needs further verification as only two low-quality clinical trials were included in this review.
\end{abstract}

Keywords: Compound salvia miltiorrhiza; Retinal vein occlusion; Systematic review

Retinal vein occlusion (RVO) is a chronic eye disease due to local obstruction of venous return. It is a common retinal vascular disease that has a serious impact on the patients' visual function. Generally, RVO patients may present with symptoms of venous tortuosity, dilatation, retinal hemorrhage, edema and exudation. It is a disease of rapid onset and long duration. Patients suffering from RVO are also at high risk of becoming blind. Similar symptoms were formulated in traditional Chinese medicine as "sudden blindness" or "blurred vision". According to statistics, RVO is ranked the second most prevalent potentially blinding retinal vascular disease after diabetic retinopathy $[1,2]$.

The disease typically affects people of over 50 years old [3]. No significant gender difference was observed. In Liu et al. 2007 report [4], she collected and analyzed the color fundus photographs of Beijing citizens after mydriasis and found that $1.3 \%$ of the population 45 years or higher was RVO patients. RVO is also believed to have led to $4.5 \%$ of the cases of monocular amblyopia and $2.1 \%$ blinding cases of the total number in her study. And its prevalence increases with age, but seems irrelevant with gender or urban-rural difference.

The pathogenesis of RVO is complex. But it is generally considered to be closely related to changes in the vessel wall, blood rheology and hemodynamics [5]. Patients may often present with cystoid macular edema and angiogenes at later stages. Some even became blind due to severe complications. There is still no causal therapy for this disease [6]. Patients are recommended to use anticoagulant and fibrinolytic drugs in the early stages. For example, western medicine practitioners use antithrombotic drug such as urokinase and streptokinase to activate plasminogen and dissolve the fibrin in blood vessels [7]. However, these drugs' effect for long-term visual improvement remains to be investigated [8]. Also, they may cause serious side effects such as intracranial and intestinal bleeding. Nowadays such a therapy is less popular. Instead, experts at home and abroad begin to turn to fundus laser photocoagulation therapy. Its effect in preventing retinal neovascularization and reducing macular edema is certain, but its use for central vision preservation or improvement is limited. Also, patients are liable to complications after this treatment $[9,10]$. Controversy continues to surround curative opportunity $[11,12]$. Intravitreally injected drugs, such as triamcinolone acetonide (TA) can also preventing retinal neovascularization and reducing macular edema, it is an invasive treatment and is reported after intravitreal injection of TA about $20 \%$ to $40 \%$ of patients will be increased intraocular pressure[13-15], some patients need repeat injections to maintain the efficacy [16].

*Corresponding author: Hongcai Shang, Evidence-based Medicine Cen tre, Tianjin University of Traditional Chinese Medicine, Nankai District, An shan Xi Dao Number 312, Tianjin 300193, China, Tel: +86-22-23051323, E-mail: shanghongcai@foxmail.com

Received October 31, 2011; Accepted February 22, 2012; Published February 26, 2012

Citation: Wang H, Shang H, Zhang J, Mu W (2012) Compound Salvia Miltiorrhiza Preparation for the Treatment of Retinal Vein Occlusion: A Systematic Review. J Diabetes Metab 3:176. doi:10.4172/2155-6156.1000176

Copyright: (c) 2012 Wang $\mathrm{H}$, et al. This is an open-access article distributed unde the terms of the Creative Commons Attribution License, which permits unrestricted use, distribution, and reproduction in any medium, provided the original author and source are credited. 
According to traditional Chinese medicine theories, retinal vein occlusion is caused by stagnation of the blood. Therefore, TCM practitioners use herbal medicine which can activate blood circulation and remove blood stasis to treat RVO patients and have gained some experiences. Such a therapy has been proved especially effective in promoting the resorption of retinal hemorrhage, reducing retinal hypoxia, alleviating retinal edema, improving blood rheology and microcirculation and recovering the visual function. Compound salvia miltiorrhiza preparation is one of these therapies. It can activate blood circulation by removing blood stasis, regulate the qi and awaken one from unconsciousness as well as clear blood vessels for better circulation. The formula also serves to nourish the blood, promote the absorption of blood stasis and inhibit angiogenesis and tissue hyperplasia. Therefore, it has a certain therapeutic effect on vision impairment caused by RVO. Furthermore, it has been proven in clinical practice that compound salvia miltiorrhiza preparation can promote the formation of collateral circulation. Ultrastructural studies also revealed that it can significantly reduce morphology injury caused by ischemia-reperfusion and improve microcirculatory blood flow. Despite all these, the efficacy and safety of this treatment remains to be evaluated. The purpose of this study is to evaluate the clinical effects and safety of compound salvia miltiorrhiza preparation in treating $\mathrm{RVO}$ and provide evidence for clinical decision-making.

\section{Methods}

\section{Inclusion criteria}

In August 2011, we did a comprehensive search for all of randomized controlled trials (RCTs) on compound salvia miltiorrhiza preparation in prevention and treatment of RVO, completed or ongoing, published or unpublished. Our search made no restrictions on language. Quasirandomized controlled trials (quasi-RCT) and controlled clinical trials (CCT) will also be included if RCTs are few in number. Cases of adverse reactions were recorded and discussed in our report.

All patients taking part in the trials were sufferers of retinal vein occlusion. They were diagnosed with RVO according to clearly defined criteria. Patients with severe organic diseases and other complications were excluded. The intervention in the treatment group was compound salvia miltiorrhiza preparation. The course of treatment was more than one week. In the two studies, comparison was made between the following five couples of intervention. 1. Compound salvia miltiorrhiza preparation V.S. placebo; 2. Compound salvia miltiorrhiza preparation V.S. anticoagulant therapy; 3. Compound salvia miltiorrhiza preparation combined with laser photocoagulation V.S. laser treatment; 4. Compound salvia miltiorrhiza preparation combined with anticoagulation treatment V.S. anticoagulation treatment; 5. Compound salvia miltiorrhiza preparation V.S. other therapies.

The primary indicator is the rate of blindness. Secondary indicators include improvement in fundus hemorrhage, reduced edema, improvement of visual function and quality of life. Incidence of adverse events was also noted. Outcome measures include at least one of the above indicator

\section{Literature search, data collection and assessment}

In August 2011, we searched the following electronic databases: the Cochrane Library, Medline-OVID, Embase-OVID, Chinese Biomedical Database (CBM), China National Knowledge Infrastructure (CNKI) Database, the Data Bank of Chinese Scientific Journals (VIP), and the Wanfang Digital Library. We developed a search strategy using terms like "retinal vein occlusion", "compound salvia droplet pill", "Danshen" and other alternative expressions in English, and in
Chinese. We also hand searched many journals of ophthalmology. And we looked through the reference part of some clinical trial reports and reviews for possible inclusion of related studies.

Study selection and data extraction were finished by two reviewers independently, both with a predefined data extraction form at hand. We also checked the result to avoid selection bias. Two reviewers assessed the quality of the included studies according to the standard described in the Cochrane Handbook [17] independently and exchanged their results. Any disagreement will be resolved by discussion.

\section{Data analysis}

We used Revman 4.2 for data analysis.Relative risk (RR) was used as a statistical index for quantitative data and weighted mean difference (WMD) for qualitative data, both with $95 \%$ confidence interval (CI). The difference value of all qualitative data before and after the treatment was calculated and analyzed. If there are two or more studies with the same intervention, homogeneity test was carried out when clinical and methodology homogeneity was observed.Moreover, a standard chi-squared statistic or $\mathrm{I}^{2}$ statistic was used to evaluate heterogeneity between subgroups. A fixed-effect model was adopted for data analysis if no statistical heterogeneity was observed between subgroups $(25 \%<$ $\left.\mathrm{I}^{2}<50 \%\right)$. In the presence of heterogeneity $\left(\mathrm{P}<0.1, \mathrm{I}^{2}>50 \%\right)$ possible causes for heterogeneity were examined. Descriptive analysis was given if no such cause can be found. Subgroup and sensitivity analysis will be carried out if sufficient studies are included. And a funnel plot will be drawn to check the existence of publication bias.

\section{Results}

\section{An overview of included studies}

Our search strategy mentioned above identified a total of 312 potentially relevant studies. After screening the title and abstract, we excluded 230 articles which are duplicates, animal experiments, case reports or observations. The full-texts of potentially relevant articles were then retrieved for more detailed assessment. Finally, two studies [18-19] on compound salvia miltiorrhiza preparation for the treatment of RVO were considered eligible for inclusion (one of them is controlled clinical trial). The selection process is shown in Figure 1.

The two studies involved 137 patients with RVO (79 in the treatment group and 58 in the control group). The median age of the 137 patients is 50. Detailed information of the included studies is shown in Table 1.

Both studies provided full diagnostic criteria, but they didn't give necessary description of the exclusion and inclusion criteria. Both mentioned that the two groups had similar baseline information and

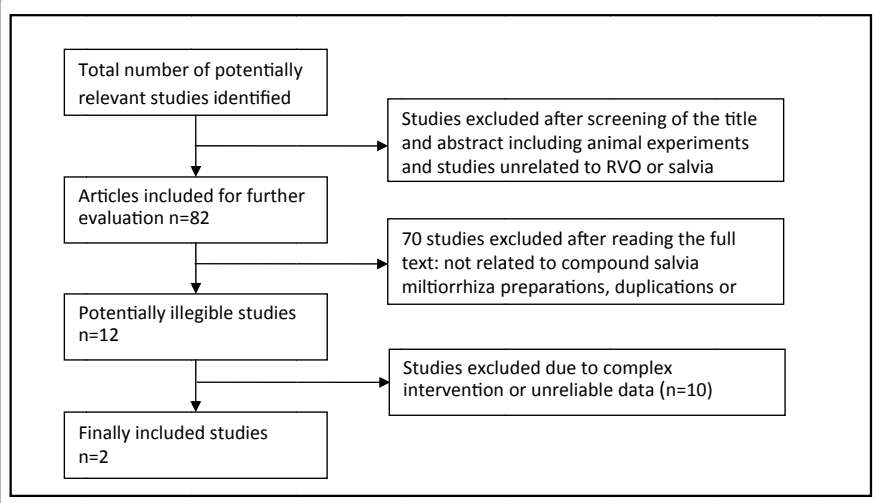

Figure 1: Flow chart of the study selection process. 
Citation: Wang H, Shang H, Zhang J, Mu W (2012) Compound Salvia Miltiorrhiza Preparation for the Treatment of Retinal Vein Occlusion: A Systematic Review. J Diabetes Metab 3:176. doi:10.4172/2155-6156.1000176

Page 3 of 5

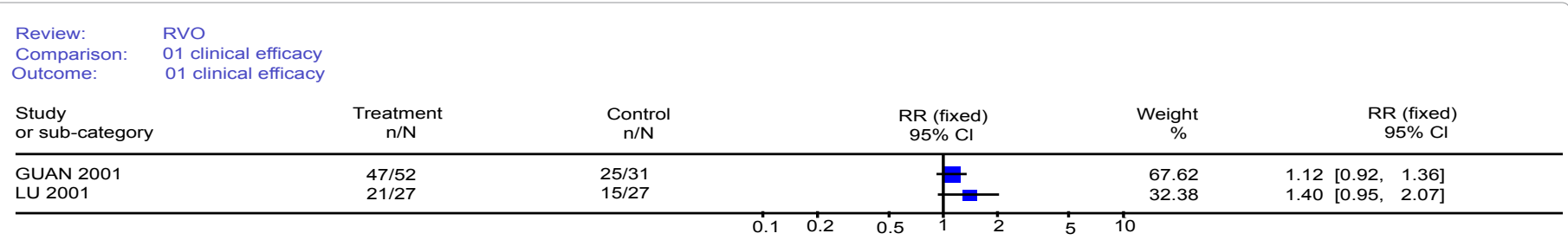

Figure 2: Result of the analysis of the therapeutic effects of compound salvia miltiorrhiza preparation in treating RVO.

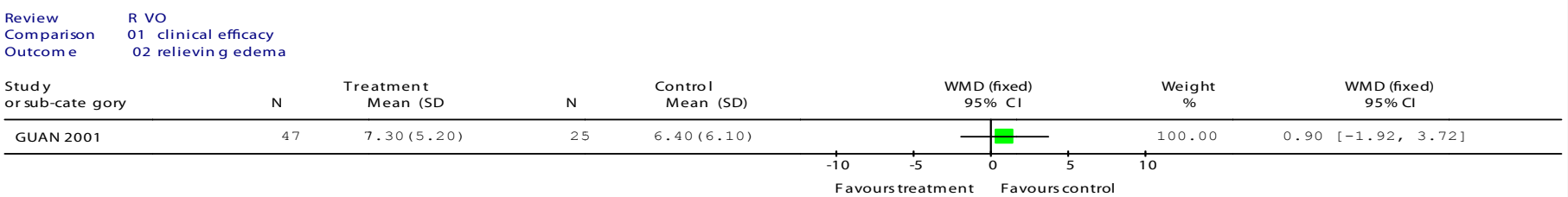

Figure 3: Result of analysis of the effect of compound salvia miltiorrhiza preparation in relieving edema in RVO patients.

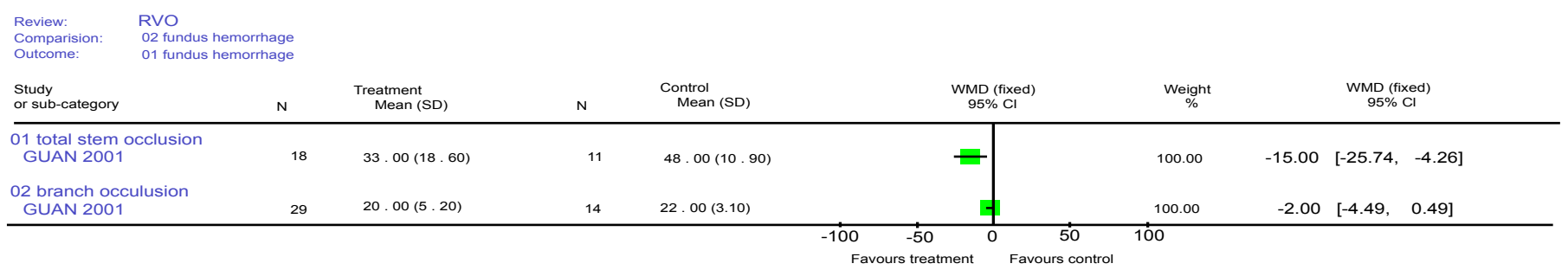

Figure 4: Result of the analysis of the therapeutic effect of compound salvia miltiorrhiza preparation in alleviating fundus hemorrhage.

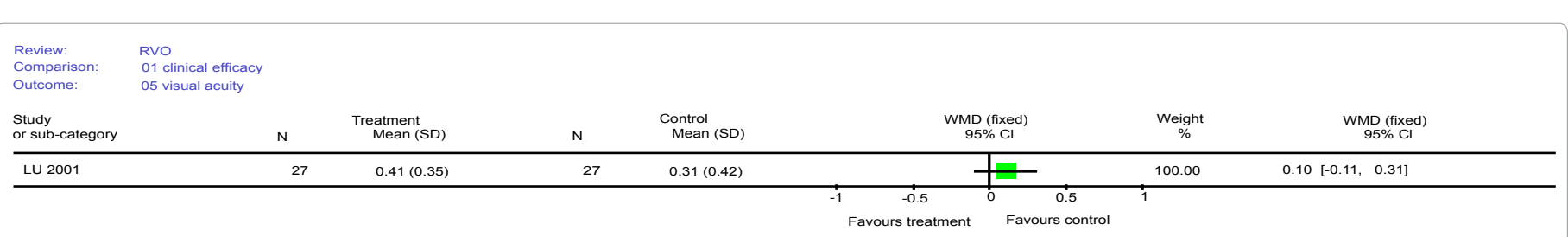

Figure 5: Result of the analysis of the effect of compound salvia miltiorrhiza preparation in improving visual acuity in RVO patients.

\begin{tabular}{|c|c|c|c|c|c|c|}
\hline $\begin{array}{l}\text { Author } \\
\text { (year) }\end{array}$ & NT/NC & $\begin{array}{l}\text { Average age } \\
\text { of patients }\end{array}$ & Treatment intervention & Control intervention & $\begin{array}{l}\text { Course of } \\
\text { treatment }\end{array}$ & Observational indicators \\
\hline $\begin{array}{l}\text { Yiqin Guan } \\
(2001)\end{array}$ & $52 / 31$ & $\begin{array}{l}\mathrm{T}: 50 \pm 12 \\
\mathrm{C}: 51 \pm 13\end{array}$ & $\begin{array}{l}\text { Compound salvia dripping } \\
\text { pills, } 15 \text { pills per time, TID }\end{array}$ & $\begin{array}{l}5000 \sim 10000 U \\
\text { urokinase added into } \\
500 \mathrm{ml} 5 \% \text { glucose } \\
\text { injection } \\
\text { iv. gtt. QD }\end{array}$ & $\begin{array}{l}\text { 30days- } \\
1 \text { year }\end{array}$ & $\begin{array}{l}\text { Therapeutic effects, reduced retinal edema, } \\
\text { relieved symptoms of fundus hemorrhage (days } \\
\text { needed for recovering from total stem occlusion/ } \\
\text { branch occlusion) }\end{array}$ \\
\hline $\begin{array}{l}\text { Junxia Lu } \\
(2001)\end{array}$ & $27 / 27$ & $\begin{array}{l}\mathrm{T}: 49.7 \pm 8.7 \\
\mathrm{C}: 51.6 \pm 7.9\end{array}$ & $\begin{array}{l}\text { 4 8ml compound salvia } \\
\text { injection } \\
\text { plus } 250 \mathrm{ml} 5 \% \text { glucose } \\
\text { injection iv. gtt.QD } \\
10 \text { times a course }\end{array}$ & $\begin{array}{l}\text { Taohong Siwu } \\
\text { Decoction } \\
\text { BID }\end{array}$ & $\begin{array}{l}3 \text { courses at } \\
\text { most }\end{array}$ & $\begin{array}{l}\text { Therapeutic effects, comparison of course of } \\
\text { disease and of treatment, improvement of visual } \\
\text { acuity after treatment, the relationship between } \\
\text { the obstructive position and therapeutic effect, } \\
\text { comparison of the onset time }\end{array}$ \\
\hline
\end{tabular}

Table 1: General characteristics of included studies.

compared the number of patients, their age and the course of disease. One study [6] has set a clear standard to judge efficacy.

Both quantitative and qualitative indicators were used in the two studies. The former include clinical effective rate and the latter include days needed for recovering from total stem blockage or from branch occlusion, logarithmic visual acuity before and after treatment and others. No cases of adverse effects were reported in either study.

\section{Methodological quality evaluation of included studies}

During literature review we found that information on study design and methodology was scarcely reported. This made it harder for us to judge the quality of included studies. Only one RCT was described as randomized, yet no words about the randomization method, allocation concealment, the use of blinding and loss of cases were mentioned. Neither were specific measures to ensure compliance. As a result, it was graded $\mathrm{C}$ according to the Cochrane standard. Analysis of the therapeutic effect of compound salvia
miltiorrhiza preparation in treating RVO

Clinical efficacy: Both studies observed the general efficacy of 
compound salvia miltiorrhiza preparation in treatment of RVO. Here, an intervention is regarded effective if improved vision, evident absorption of fundus hemorrhage or shortened venous filling time revealed by fundus fluorescein angiography was reported after the treatment. On the other hand, an intervention is defined ineffective if no significant improvement or a decline in the patients' vision, ongoing or worsened fundus hemorrhage or no change or deterioration of symptoms revealed by fundus fluorescein angiography was observed in the patients receiving the treatment

In both studies, clinical efficacy indicators which categorize the treatment efficacy as effective (visible or effective) or ineffective (invalid or worse) were used to analyze the results. The results from the two studies cannot be pooled because of obvious heterogeneity (RR 1.12, $95 \% \mathrm{CI}[0.92,1.36]$ and RR $1.40,95 \% \mathrm{CI}[0.95,2.07], \mathrm{P}=0.04)$. The results showed there was no significant difference between the two groups.

\section{Improvement of visual functions and fundus}

Days needed for recovery from retinal edema: One study reported the effect of compound salvia injection in alleviating edema in RVO patients. The results showed there was no significant difference between the two groups (WMD 0.90, 95\%CI [- 1.92, 3.72], $\mathrm{P}=0.53$ )

Days needed for recovering from fundus hemorrhage (total stem occlusion/branch occlusion): In one study, the time needed for RVO patients to recover form fundus hemorrhage and edema was used as a measure to evaluate the effectiveness of the intervention. Analysis of the data for the indicator "time needed for total stem blockage recovery" demonstrated that compound salvia injection has a better effect than Urokinase (WMD - 15, 95\%CI [- 25.74, - 4.26], $\mathrm{P}=0.006$ ). However, there was no significant difference in the effectiveness of the two interventions in relieving branch occlusion that can be concluded from the data analysis of the indicator "days needed for branch occlusion recovery" (WMD -2, 95\%CI [- 4.49, 0.49], P = 0.12).

\section{Improvement in visual acuity}

One study observed the effect of compound salvia decoction in improving the visual function of RVO patients. No significant statistical difference was found between the two groups (WMD 0.10, $95 \% \mathrm{CI}[-0.11,0.31], \mathrm{P}=0.34)$.

\section{Safety analysis}

In the included studies, no case of adverse effects of compound salvia miltiorrhiza preparation in treatment of RVO patients was reported.

\section{Discussion}

The results of our systematic review of the included studies and quantitative analysis of data suggested that there was no significant difference between the effectiveness of compound salvia miltiorrhiza preparation and that of the control intervention in treatment of RVO. So far a definite conclusion cannot be drawn.

According to the Cochrane Handbook 4.2.6, we evaluated the quality of individual studies included in the systematic review. We found that one study has methodological problems, especially in the use of randomization and blinding. This RCT is graded C. We also found other problems with the two studies. For instance, the inclusion and exclusion criteria were not clearly defined. No criteria for patient admission and dropouts were established. And the baseline information (age, sex ratio, course of disease, conditions and co morbidity) was not completely consistent. All these may lead to obvious selection bias. No study used a simulated agent. As the control interventions were different, it is difficult to avoid performance bias.

Moreover, relevant endpoint indicators (such as blinding rate) were not reported in either study. Information on the patients' life quality and of their follow-up was also lacking. Consequently, we cannot make a conclusion concerning these important outcomes.

We cannot draw a definite conclusion from this systematic review as the number of RCTs we included was small and both were low in quality. But we will continue to upgrade this study. And in this process our inclusion criteria for selection of RCTs will be tightened.

\section{Conclusion}

\section{Suggestions for clinical practice}

As only two studies of low quality were included in this review, a conclusion on the efficacy and safety of the formula cannot be safely made. More well-designed, strictly-executed RCTs with a large sample and adequate follow-up are needed to provide better evidence for the use of compound salvia miltiorrhiza preparation in treatment of RVO.

\section{Suggestions for clinical research}

We noticed that the quality of clinical studies needs to be improved. A clinical study should have a scientific and standardized design. Here, a sound top-level devising is essential for a good clinical research project. Other aspects such as random distribution, use of blinding and data analysis also deserve high attention. With respect to outcomes, it is recommended that endpoint measures that bear a close relationship with the condition of patients be used. Such indicators include mortality rate, incidence rate and quality of life. Moreover,long-term follow-up is recommended for better observation of the long-term effects of the treatment and its effects on prognosis.

\section{Acknowledgement}

Contract/grant sponsor: National Science and Technology Major Project of China

Contract/grant number: 2012ZX09303010-001

Contract/grant sponsor: Program for New Century Excellent Talents in University

Contract/grant number: NCET-09-0900

\section{References}

1. Hua-ping Liao, Ling Wang (2005) Advances in therapy of retinal vein occlusion International Journal of Ophthalmology 5: 537-542.

2. Xiaoqin Lei, Pengcheng He, Mingfang Wang (2002) Status Quo Researches on Neovascularization as a Complication of Retinal Vein occlusion. US Chinese Journal of Ophthalmology 2: 28-30.

3. Mitchell P, Smith W, Chang A (1996) Prevalence and associations of retinal vein occlusion in Australia.The Blue Mountains Eye Study. Arch Ophthalmo 114: 1243-1247.

4. Liu Wei-wei, Xu Liang, Xie Xi-wei (2007) Epidemiology of retinal vein occlusion in adult Chinese population in rural and urban areas in Beijing. Ophthalmol CHN 16: 233-236

5. Fengming LI (1996) Pandect of ophthalmology. Peking: People's Medical Publishing House.

6. Yang Wen-Zhi, Wu Qiang (2010) Advances on treatment for retinal vein occlusion. Rec Adv Ophthalmol 30: 694-697

7. Jiang Hong, Feng Zhen-e (2009) Pathogenesis and treatment of retinal vein occlusion. Journal of Ningxia Medical University 3: 692-694.

8. Hayreh SS (2005) Prevalent misconceptions about acute retinal vascular occlusive disorders. Prog Retin Eye Res 24: 493-519. 
Citation: Wang H, Shang H, Zhang J, Mu W (2012) Compound Salvia Miltiorrhiza Preparation for the Treatment of Retinal Vein Occlusion: A Systematic Review. J Diabetes Metab 3:176. doi:10.4172/2155-6156.1000176

Page 5 of 5

9. Ouyang Yun, Cao Shuxia, Zhang Jian (2010) Advances of the treatment of retinal vein occlusion. Int J Ophthalmol 10: 1937-1939.

10. Hayreh SS, Rubebstein L, Podhajsky P (1993) Argon laser scatter photocoagulation in treatment of branch retinal vein occlusion. A prospective clinical trial. Ophthalmologica 206: 1-14.

11. Rong Liang, Ren Dayuan, Lu Bingchen (2011) $532 \mathrm{~nm}$ argon laser photocoagulation for diabetic retinopathy. Practical Journal of Medicine \& Pharmacy 28: 216-217.

12. Rong Hu, Ling Gong, De-Yong Jiang (2011) Research on intravitreal Bevacizumab combined with retinal photocoagulation for iris and retinal neovascularization. International Journal of Ophthalmology 11: 485-487.

13. Jonas JB (2006) Intravitreal triamcinolone acetonide: A change in paradigm. Ophthalmic Res. 38: 218-245

14. Jonas JB, Degenring RF, Kreissig I, Akkoyun I, Kamppeter BA (2005)
Intraocular pressure elevation after intravitreal triamcinolone acetonide injection. Ophthalmology 112: 593-598.

15. Shukla D, Vidhya N, Prasad NM, Mahalakshmi R, Kolluru C, et al. (2007) Evaluation of patient age as a risk factor for intraocular pressure elevation after intravitreal triamcinolone. Am J Ophthalmol 144: 453-454.

16. Cekic O, Chang S, Tseng JJ, Barile GR, Del Priore LV, et al. (2005) Intravitreal triamcinolone injection for treatment of macular edema secondary to branch retinal vein occlusion. Retina 25: 851-855.

17. The Cochrane Collaboration (2006) Cochrane Handbook for Systematic Reviews of Interventions 4.2.6. UK, The Cochrane Collaboration.

18. Yiqin Guan (2001) Clinical observation on the treatment of central retinal vein occlusion with Compound Salvia droplet pill. Tianjin pharmacy 13: 54-55.

19. Junxia Lu, Hui Pei, Jianying Song (2001) Clinical observation on the treatment of retinal vein obstruction with Compound Salvia injection. Science and technology of traditional chinese medicine 8: 122. 